Fragen zur GOÄ-Abrechnung
Kostenloser Leserservice!

Haben Sie Fragen zur privatärztlichen Abrechnung? Antworten gibt unser Experte Dr. med. Helmut Hoffmann, München.

Schreiben Sie an: Urban \& Vogel GmbH Redaktion HNO-NACHRICHTEN Aschauer Straße 30 81549 München Fax 0892030431400 E-Mail: gabriele.zoerrgiebel @springer.com

\title{
Bei einer Mutter und ihren drei Kindern sollte ich die Gehörgänge medizinisch reinigen. Ich berechnete nach GOÄ jeweils 1415 2x; 1565; 5; 1. Die Mutter in- formierte die Abrechnungsstelle, sie könne den Leistungsumfang nicht nach- vollziehen. Ist die Liquidation berechtigt ?
}

\section{Antwort:}

GOÄ-Nr 1 „Beratung, auch mittels Fernsprecher - (neben Leistungen des Abschnittes $\mathrm{C}$ bis $\mathrm{O}$ nur $1 \mathrm{x}$ im Behandlungsfall) -80 Punkte“. Der Begriff der "Beratung“ in der Gebührenordnung ist umfassend und schließt z.B. folgende Teilleistungen ein:

— Anhören der Beschwerden bzw. gezielte Befragung nach den Beschwerden

- Aufnahme der Krankheitsvorgeschichte soweit nicht hierfür gesondert berechnungsfähige Anamneseleistungen ausgewiesen sind

_Erteilung von Auskünften (Aufklärung, Information, Belehrung etc.)

- Besprechung von Verhaltensmaßnahmen

- Beantwortung von spezifischen Fragen des Patienten

— Erläuterung der beabsichtigten diagnostischen und/oder therapeutischen Maßnahmen

_ Ausstellung von Verordnungen oder Überweisungen.

Der Umfang der Beratung wird vom Arzt im Einzelfall nach pflichtgemäßem Ermessen bestimmt und hat der jeweils gegebenen medizinischen Situation zu entsprechen. Die Beratung richtet sich primär unmittelbar an den Kranken. Allerdings gibt es eine Reihe von Situationen, in denen der Kranke nur mittelbar, d.h. über eine Betreuungsoder Bezugsperson beraten wird. Dies kann z.B. der Fall sein im Zusammenhang mit der
Behandlung von Kindern, von Bewusstlosen oder von psychisch Kranken.

Wenn die dargestellten Minimalanforderungen zur Abrechnung der Nr. 1 GOÄ tatsächlich bei jedem der vier Patienten individuell erbracht wurden, ist der Ansatz der Beratungsleistung nach Nr. 1 bei jedem einzelnen Patienten gebührenrechtlich nicht zu beanstanden. Die GOÄ sieht keinen Abschlag oder eine Nicht-Abrechnungsfähigkeit der Nr. 1 bei Behandlung mehrerer Familienmitglieder während einer familiären Arzt-Patienten-Begegnung vor, wenn die gebührenrechtlichen Voraussetzungen für den Ansatz der Nr. 1 GOÄ gegeben sind.

Da es sich bei der Mutter und ihren drei unmündigen Kindern jedoch um dieselbe Diagnose (Verstopfung der Gehörgänge durch (erumen) handelte, und diese von vorneherein feststand, kann man davon ausgehen, dass die Beratung sich gleichlautend in Hinweisen auf die allgemeine Ohrhygiene und die Erklärung der therapeutischen Maßnahmen erschöpfte. Weiterhin richteten sich die Beratungsinhalte in allen vier Fällen vermutlich an die Mutter. Die Beratung erfolgte im Falle der Kinder nur mittelbar, d.h. über die Betreuungs- oder Bezugsperson.

Nachdem der Umfang der Beratung vom Arzt im Einzelfall nach pflichtgemäßem Ermessen bestimmt werden soll und der jeweils gegebenen medizinischen Situation zu entsprechen hat, wird aus medizinischer Sicht - aber ohne gebührenrechtliche Grundlage - vorgeschlagen, im Falle der drei Kinder die Nr. 1 GOÄ mit dem einfachen Gebührensatz zu berechnen. Ein Wegfall der Nr. 1 GOÄ erscheint in der oben vorgetragenen Konstellation nicht sachgerecht, da sich der behandelnde HNO-Arzt mit jedem einzelnen Kind und dessen Beschwerden individuell befasst hat.

Die GOÄ-Nr. 5: „Symptombezogene Untersuchung - 80 Punkte" wurde in allen 4 Fällen erbracht und kann deshalb auch in Rechnung gestellt werden. Die Zahlungsverweigerung ist hier völlig unbegründet!

GOÄ-Nr. 1415 (2x): „Binokularmikroskopische Untersuchung des Trommelfells und/oder der Paukenhöhle zwecks diagnostischer Abklärung, als selbständige Leistung - 91 Punkte“. GOÄ-Nr. 1415 kann selbstverständlich für jedes Ohr getrennt angesetzt werden. Es handelt sich jedoch um eine diagnostische Leistung und nicht um eine Führungshilfe für eine Therapie, z. B. Granulations- oder Cerumenentfernung, mikrochirurgische Operation. Wenn die mikroskopische Diagnostik der Ohren nach erfolgter Reinigung des Gehörgangs gemäß GOÄ-Nr. 1565 „Entfernung von obstruierenden Ohrschmalzpfröpfen, auch beidseitig - 45 Punkte" durchgeführt und dafür in Rechnung gestellt wurde, ist gegen den Ansatz der Nr. 1415 nichts einzuwenden. 